\title{
Coherent Control of Collisional Events: Bimolecular Reactive Scattering
}

\author{
Moshe Shapiro \\ Department of Chemical Physics, The Weizmann Institute of Science, Rehovot 76100, Israel \\ Paul Brumer \\ Chemical Physics Theory Group, Department of Chemistry, University of Toronto, Toronto, Canada M5S3H6
}

(Received 6 September 1995)

\begin{abstract}
Laser control over reactive and nonreactive collisional events of the $B+C \rightarrow F+G$ type is shown possible via preparation of a superposition of degenerate initial states which interfere with one another. Required, however, is a controlled superposition of continuum states comprised of correlated products of internal $B, C$ states and plane waves. We show that this can be achieved by the controlled preparation of a $B$ superposition state followed by a shift in the kinetic energy of the superposition state components, guaranteeing the required degeneracy of terms in the superposition state and a reduction in center of mass related oscillations. [S0031-9007(96)01146-5]
\end{abstract}

PACS numbers: 82.40.Dm, 34.50.Rk, 34.50.Lf

The essence of coherent control of molecular processes is to arrive at the final state via two or more independent paths which can quantum mechanically interfere. By varying laboratory parameters one directly alters this interference giving active control over the final state. This approach has been successfully demonstrated, both theoretically [1-4] and experimentally [5-8], in unimolecular breakdown reactions, such as photodissociation [8] and photoionization [6]. However, the issue of effectively controlling collisional events has, despite some effort [9], thus far remained unresolved.

In this Letter we develop coherent control for collisional processes. In particular, we show that creating an initial state comprised of a superposition of degenerate Hamiltonian eigenstates with correlated molecular and translational states allows for control over the reaction products. However, we experimentally realizing such correlated states presents a serious challenge. We propose one resolution to this problem by demonstrating that such a superposition can be produced via an initial preparatory step for one reactant followed by a specific choice of reactant collision velocity. The result is the extension of coherent control to the entire class of collisional processes.

Consider the collision of a beam of molecules $B$ with a beam of molecules or atoms $C$, which yields products $F$ and $G$, i.e.,

$$
B+C \longrightarrow F+G .
$$

$F$ and $G$ can be identical to (nonreactive scattering), or different from (reactive scattering) $B$ and $C$. We denote $B+C$ as the $\beta$ arrangement and $F+G$ as the $\gamma$ arrangement. Traditional time independent scattering theory proceeds by considering Eq. (1) with $B+C$ starting in an eigenstate of the free Hamiltonian $H_{\beta}^{0}$ in the $\beta$ arrangement,

$$
H_{\beta}^{0}=K_{\beta}+h_{B}+h_{C},
$$

with $K_{\beta}$ being the kinetic energy of the $B-C$ relative motion and $h_{B}, h_{C}$ denoting the internal Hamiltonians of $B$ and $C$.

To attain control over the process consider then the following superposition, which we show below can be realized experimentally, as the initial asymptotic state,

$$
|n, \beta\rangle=|0, C\rangle \sum_{i=1,2} a_{i}|i, B\rangle\left|E_{\beta}^{\mathrm{kin}}(i)\right\rangle\left|E_{\mathrm{cm}}(i)\right\rangle .
$$

Here

$$
E_{\beta}^{\mathrm{kin}}(i)=E-\varepsilon_{C}(0)-\varepsilon_{B}(i),
$$

where $|i, X\rangle$ with $X=B, C$ are eigenstates, of energy $\varepsilon_{X}(i)$, of $h_{B}$ and $h_{C}$,

$$
\left[\varepsilon_{X}(i)-h_{X}\right]|i, X\rangle=0, \quad X=B, C .
$$

The $\left|E_{\beta}^{\mathrm{kin}}(i)\right\rangle$ and $\left|E_{\mathrm{cm}}(i)\right\rangle$ states are plane waves describing the free motion of $B$ relative to $C$, and the motion of the $B-C$ center of mass, i.e., $\left\langle\mathbf{r}_{B C} \mid E_{\beta}^{\mathrm{kin}}(i)\right\rangle=$ $\exp \left(i \mathbf{k}_{i} \cdot \mathbf{r}_{B C}\right),\left\langle\mathbf{R}_{B C} \mid E_{\mathrm{cm}}(i)\right\rangle=\exp \left(i \mathbf{K}_{i} \cdot \mathbf{R}_{B C}\right)$, where $\left|\mathbf{k}_{i}\right|=\left\{2 \mu_{B C} E_{\beta}^{\mathrm{kin}}(i)\right\}^{1 / 2} / \hbar, \mu_{B C}=m_{B} m_{C} /\left(m_{B}+m_{C}\right)$ is the reduced mass of the $B C$ pair, and $\mathbf{K}_{i}$ is the $B C$ center of mass momentum. Here $\mathbf{R}_{B C}, \mathbf{r}_{B C}$ are the position of the $B C$ center of mass and the $B-C$ relative vector, respectively.

The superposition state $|n, \beta\rangle$ is composed of degenerate eigenstates of $H_{\beta}^{0}$,

$$
\left[E-H_{\beta}^{0}\right]|0, C\rangle|i, B\rangle\left|E-\varepsilon_{C}(0)-\varepsilon_{B}(i)\right\rangle=0,
$$

and center of mass terms. As a result, we can use standard time-independent scattering theory [10] to calculate the cross section for scattering from this state to any of the $\gamma$-arrangement final states. The latter states, of the form $|j, \gamma\rangle\left|E^{\mathrm{kin}}\right\rangle\left|E_{\mathrm{cm}}(i)\right\rangle$, have a component in the center of 
mass system which satisfies the free Schrödinger equation in the product space,

$$
\left[E-H_{\gamma}^{0}\right]|j, \gamma\rangle\left|E-\varepsilon_{\gamma}(j)\right\rangle=0,
$$

where $|j, \gamma\rangle$ are the eigenstates of the $F+G$ internal Hamiltonians,

$$
\left[\varepsilon_{\gamma}(j)-h_{F}-h_{G}\right]|j, \gamma\rangle=0,
$$

and $H_{\gamma}^{0}=K_{\gamma}+h_{F}+h_{G}$ is analogous to Eq. (2), describing the product in arrangement $\gamma$.

The cross section for forming one of the $\gamma$-arrangement final states, having started from the $|n, \beta\rangle$ superposition state, is given by

$$
\sigma(j, \gamma \longleftarrow n, \beta \mid E)=\mid \sum_{l=1,2}\left\langle E_{\mathrm{cm}}(l)\left|\left\langle E, j, \gamma^{-}\left|V_{\beta}\right| n, \beta\right\rangle\right|^{2} .\right.
$$

Here $V_{\beta}=H-H_{\beta}^{0}$ is the (reactive or nonreactive) interaction potential, with $H$ being the Hamiltonian in the center of mass system. $\left|E, j, \gamma^{-}\right\rangle$are incoming eigenstates of $H$,

$$
[E-H]\left|E, j, \gamma^{-}\right\rangle=0
$$

which go over in the asymptotic limit to a specific free state of the $F+G$ products,

$$
\begin{aligned}
\exp (-i E t / \hbar)\left|E, j, \gamma^{-}\right\rangle & \stackrel{t \rightarrow \infty}{\longrightarrow} \exp (-i E t / \hbar) \\
& \times\left|E-\varepsilon_{y}(j)\right\rangle|j, \gamma\rangle .
\end{aligned}
$$

Substituting Eq. (3) into Eq. (9) gives, for the reactive cross section,

$$
\begin{aligned}
\sigma(j, \gamma \longleftarrow n, \beta \mid E) & \left.=\left|\sum_{l=1,2}\left\langle E_{\mathrm{cm}}(l)\left|\sum_{i=1,2} a_{i}\left\langle E, j, \gamma^{-}\left|V_{\beta}\right| 0, C\right\rangle\right| i, B\right\rangle\right| E_{\beta}^{\mathrm{kin}}(i)\right\rangle\left.\left|E_{\mathrm{cm}}(i)\right\rangle\right|^{2} \\
& =\left|a_{1}\right|^{2} \sigma_{11}^{R}(j)+\left|a_{2}\right|^{2} \sigma_{22}^{R}(j)+2 \mathrm{R}_{e} a_{1}^{*} a_{2} \sigma_{12}^{R}(j), \quad \gamma \neq \beta,
\end{aligned}
$$

where

$$
\begin{aligned}
\sigma_{i i}^{R}(j)= & \left.\left|\left\langle E, j, \gamma^{-}\left|V_{\beta}\right| 0, C\right\rangle\right| E_{\beta}^{\mathrm{kin}}(i)\right\rangle\left.\right|^{2}, \quad i=1,2, \\
\sigma_{12}^{R}(j)= & \left\langle E_{\beta}^{\mathrm{kin}}(1)\right|\langle 1, B|\left\langle 0, C\left|V_{\beta}\right| E, j, \gamma^{-}\right\rangle \\
& \times\left\langle E, j, \gamma^{-}\left|V_{\beta}\right| 0, C\right\rangle|2, B\rangle\left|E_{\beta}^{\mathrm{kin}}(2)\right\rangle\left\langle E_{\mathrm{cm}}(1) \mid E_{\mathrm{cm}}(2)\right\rangle, \quad \gamma \neq \beta .
\end{aligned}
$$

Although the matrix element $\left\langle E_{\mathrm{cm}}(1) \mid E_{\mathrm{cm}}(2)\right\rangle=$ $\int d \mathbf{R}_{B C} \exp \left[i\left(\mathbf{K}_{2}-\mathbf{K}_{1}\right) \cdot \mathbf{R}_{B C}\right]$ integrated over all space is zero, the proper region of integration in this case is the intersection volume of the $B$ and $C$ beams. Hence if $\left(\mathbf{K}_{2}-\mathbf{K}_{1}\right)$ is made sufficiently small then the integral over this region can be made nonzero and control over the cross section is possible. An experimental means of achieving this result is discussed below.

Although Eq. (13) indicates that one can control detailed cross sections, often we only want to control the total reactive vs the total nonreactive cross section. In that case, the reactive to nonreactive branching ratio is given as

$$
\begin{aligned}
\frac{\sigma^{R}}{\sigma^{N R}} & =\frac{\sum_{j} \sigma(j, \gamma \neq \beta \leftarrow n, \beta \mid E)}{\sum_{j}(j, \beta \leftarrow n, \beta \mid E)} \\
& =\frac{\sigma_{11}^{R}+x^{2} \sigma_{22}^{R}+2 x\left|\sigma_{12}^{R}\right| \cos \left(\delta_{12}^{R}+\theta_{12}\right)}{\sigma_{11}^{N R}+x^{2} \sigma_{22}^{N R}+2 x\left|\sigma_{12}^{N R}\right| \cos \left(\delta_{12}^{N R}+\theta_{12}\right)},
\end{aligned}
$$

where $x=\left|a_{2} / a_{1}\right|, \theta_{12}=\arg \left(a_{2} / a_{1}\right), \sigma_{i k}^{R}=\sum_{j} \sigma_{i k}^{R}(j)$, $i, k=1,2$, with similar definitions holding for $\sigma_{i k}^{N R}$, and $\delta_{12}^{R}=\arg \left(\sigma_{12}^{R}\right), \delta_{12}^{N R}=\arg \left(\sigma_{12}^{N R}\right)$. Thus, the reactive vs nonreactive cross-section ratio can be controlled by varying the relative magnitude, $x$, and the relative phase, $\theta_{12}$, of the $a_{1}$ and $a_{2}$ coefficients.

Control over the $a_{i}$ can be attained by a number of routes. One approach prepares the $B-C$ superposition by exciting $B$ to a superposition state and colliding the result with $C$. Specifically, consider preparing $|n, \beta\rangle$ by first irradiating $|1, B\rangle\left|E_{B}^{\mathrm{kin}}(1)\right\rangle$ to produce

$$
|n, B\rangle=\sum_{i=1,2} a|i, B\rangle\left|E_{B}^{\mathrm{kin}}(1)\right\rangle
$$

where $\left|E_{B}^{\mathrm{kin}}(1)\right\rangle$ describes the motion of the center of mass of $B$. We now alter the velocities of $|1, B\rangle$ and $|2, B\rangle$ to give

$$
|n, B\rangle=\sum_{i=1,2} a_{i}|i, B\rangle\left|E_{B}^{\mathrm{kin}}(i)\right\rangle
$$

where $\left\langle\mathbf{r}_{B} \mid E_{B}^{\mathrm{kin}}(i)\right\rangle=\exp \left(i \mathbf{k}_{i}^{B} \cdot \mathbf{r}_{B}\right), \quad \mathbf{k}_{1}^{B} \neq \mathbf{k}_{2}^{B}, \quad$ and where $\mathbf{r}_{B}$ is the laboratory position of $B$. This can be done, for example, by passing the superposition 
[Eq. (15)] through a hexapole field. Then, colliding the $|n, B\rangle$ superposition state with particle $C$ of momentum $\mathbf{k}^{C}$ gives the $B-C$ superposition state

$$
|\psi\rangle=|0, C\rangle \sum_{i=1,2} a_{i}|i, B\rangle\left|E_{B}^{\mathrm{kin}}(i)\right\rangle\left|E_{C}^{\mathrm{kin}}\right\rangle\left|E_{\mathrm{cm}}(i)\right\rangle .
$$

To produce Eq. (3), however, requires that the degeneracy condition [Eq. (4)] be satisfied, i.e., that

$$
E_{\beta}^{\mathrm{kin}}(1)-E_{\beta}^{\mathrm{kin}}(2)=\varepsilon_{B}(2)-\varepsilon_{B}(1) .
$$

That is, with $\mathbf{K}_{i}=\mathbf{k}_{i}^{B}+\mathbf{k}_{C}$ and with the $B-C$ relative center of mass momentum given by $\mathbf{k}_{i}=\left(m_{C} \mathbf{k}_{i}^{B}-\right.$ $\left.m_{B} \mathbf{k}_{C}\right) /\left(m_{B}+m_{C}\right)$, Eq. (18) becomes

$$
\left(\hbar^{2} / 2 \mu_{B C}\right)\left(k_{1}^{2}-k_{2}^{2}\right)=\varepsilon_{B}(2)-\varepsilon_{B}(1)
$$

or

$$
\begin{aligned}
\left\{\hbar^{2} /\left[2\left(m_{B}+m_{C}\right)\right]\right\} & \left\{\left(m_{C} / m_{B}\right)\left[\left(k_{1}^{B}\right)^{2}-\left(k_{2}^{B}\right)^{2}\right]\right. \\
& \left.-2 \mathbf{k}_{C} \cdot\left(\mathbf{k}_{1}^{B}-\mathbf{k}_{2}^{B}\right)\right\}=\varepsilon_{B}(2)-\varepsilon_{B}(1) .
\end{aligned}
$$

Thus, to achieve control requires that $\mathbf{k}_{i}^{B}, \mathbf{k}_{C}$ be chosen to satisfy the degeneracy condition imposed by Eq. (20). Further, $\left\langle E_{\mathrm{cm}}(1) \mid E_{\mathrm{cm}}(2)\right\rangle$ must be nonzero. Since, by virtue of the definition of $\mathbf{K}_{i}, \int d \mathbf{R}_{B C} \exp \left[i\left(\mathbf{K}_{1}-\mathbf{K}_{2}\right)\right.$. $\left.\mathbf{R}_{B C}\right]=\int d \mathbf{R}_{B C} \exp \left[i\left(\mathbf{k}_{1}^{B}-\mathbf{k}_{2}^{B}\right) \cdot \mathbf{R}_{B C}\right]$, if $\left(\mathbf{k}_{1}^{B}-\mathbf{k}_{2}^{B}\right)$ is made sufficiently small then $\left\langle E_{\mathrm{cm}}(1) \mid E_{\mathrm{cm}}(2)\right\rangle$ is nonzero when integrated over the volume of intersection of the $B$ and $C$ beams. Under these circumstances Eq. (20) becomes

$$
\left\{\hbar^{2} /\left(m_{B}+m_{C}\right)\right\}\left[\mathbf{k}_{C} \cdot\left(\mathbf{k}_{1}^{B}-\mathbf{k}_{2}^{B}\right)\right] \approx \varepsilon_{B}(1)-\varepsilon_{B}(2),
$$

so that large $\mathbf{k}_{C}$ may be required to satisfy this condition. With such a $\mathbf{k}_{C}$, bimolecular control, regulated by the amplitude and phases of the $a_{i}$, is established.

Alternative methods of preparing the superposition in Eq. (3) and maintaining a nonzero $\left\langle E_{\mathrm{cm}}(1) \mid E_{\mathrm{cm}}(2)\right\rangle$ can be envisioned. The most obvious deals with superposing degenerate states of $B$. The energy degeneracy requirement is then automatically satisfied and, since $\mathbf{K}_{1}=\mathbf{K}_{2}$, then $\left\langle E_{\mathrm{cm}}(1) \mid E_{\mathrm{cm}}(2)\right\rangle$ is trivially nonzero. Examples include collisions such as $\mathrm{H}(2 s)+D$ in a superposition with $\mathrm{H}(2 p)+D$, where $D$ is a molecule and where $\mathrm{H}(2 s), \mathrm{H}(2 p)$ result from a prior coherently controlled photolysis of $\mathrm{H}_{2}$. Similarly, one can envision using elliptically polarized light to prepare a superposition of $m_{j}$ states, where $m_{j}$ is the $z$ projection of the rotational angular momentum of a diatomic $B$, and then colliding the result with $C$. Once again the degeneracy of the states ensure that control is possible and that the center of mass overlap matrix element is nonzero.

Finally note that the above formalism can be readily extended to general superposition states of the form

$$
|n, \beta\rangle=\sum_{i, l} a_{i l}|i, B\rangle|l, C\rangle\left|E_{\beta}^{\mathrm{kin}}(i, l)\right\rangle\left|E_{\mathrm{cm}}(i, l)\right\rangle,
$$

with $E_{\beta}^{\mathrm{kin}}(i, l)=E-\varepsilon_{C}(l)-\varepsilon_{B}(i) . \quad$ Here, the $\left|E_{\beta}^{\mathrm{kin}}(i, l)\right\rangle$ states are plane waves describing the free motion of $B$ relative to $C\left[\left\langle\mathbf{R} \mid E_{\beta}^{\mathrm{kin}}(i, l)\right\rangle \equiv \exp \left(i \mathbf{k}_{i l} \cdot \mathbf{R}\right)\right]$, where $\left|\mathbf{k}_{i l}\right|=\left\{2 \mu_{B C} E_{\beta}^{\text {kin }}(i, l)\right\}^{1 / 2} / \hbar$. That is, we can show that such a superposition leads to interference if the $\left\langle E_{\mathrm{cm}}(j, k) \mid E_{\mathrm{cm}}(i, l)\right\rangle$ are nonzero, and hence to the possibility of control over the reaction cross sections. We are currently examining possible methods for experimentally realizing such states.

In summary, we have introduced a means of achieving coherent control of collisional processes, applicable to a wide range of phenomena including atom-atom interactions, bimolecular collisions, and nuclear reactions, and opening a new area of applications in the coherent control of molecular processes.

We wish to thank Prof. R. Bersohn for many helpful discussions and Prof. W. H. Miller for his insights into the role of the phases associated with center of mass motion. We thank the U.S. Office of Naval Research and the OLLRC for support of this work.

[1] For recent reviews see, e.g., M. Shapiro and P. Brumer, in International Reviews in Physical Chemistry (Taylor \& Francis, London, 1994), Vol. 13, p. 187; P. Brumer and M. Shapiro, Annu. Rev. Phys. Chem. 43, 257 (1992).

[2] C. Asaro, P. Brumer, and M. Shapiro, Phys. Rev. Lett. 60, 1634 (1988).

[3] G. Kurizki, M. Shapiro, and P. Brumer, Phys. Rev. B 39, 3435 (1989).

[4] M. Shapiro, J. W. Hepburn, and P. Brumer, Chem. Phys. Lett. 149, 451 (1988).

[5] C. Chen, Y-Y. Yin, and D. S. Elliott, Phys. Rev. Lett. 64, 507 (1990); 65, 1737 (1990).

[6] S. M. Park, S-P. Lu, and R. J. Gordon, J. Chem. Phys. 94, 8622 (1991); B. A. Baranova, A. N. Chudinov, and B. Ya. Zel'dovitch, Opt. Commun. 79, 116 (1990); V. D. Kleiman, L. Zhu, X. Li, and R. G. Gordon, J. Chem. Phys. 102, 5863 (1995).

[7] E. Dupont, P. B. Corkum, H.C. Liu, M. Buchanan, and Z. R. Wasilewski, Phys. Rev. Lett. 74, 3596 (1995).

[8] B. Sheeny, B. Walker, and L. F. DiMauro, Phys. Rev. Lett. 74, 4799 (1995).

[9] J. L. Krause, M. Shapiro, and P. Brumer, J. Chem. Phys. 92, 1126 (1990).

[10] R. D. Levine, Quantum Mechanics of Molecular Rate Processes (Clarendon, Oxford, England, 1969); J. R. Taylor, Scattering Theory (Wiley, New York, 1972). 